\title{
Imaging of joints with laser-based photoacoustic tomography: An animal study
}

\author{
Xueding Wang ${ }^{\text {a) }}$ \\ Department of Radiology, University of Michigan School of Medicine, Ann Arbor, Michigan 48109 \\ David L. Chamberland ${ }^{\text {b) }}$ \\ Division of Rheumatology, Department of Internal Medicine, University of Michigan School of Medicine, \\ Ann Arbor, Michigan 48109 \\ Paul L. Carson, J. Brian Fowlkes, Ronald O. Bude, and David A. Jamadar \\ Department of Radiology, University of Michigan School of Medicine, Ann Arbor, Michigan 48109 \\ Blake J. Roessler \\ Division of Rheumatology, Department of Internal Medicine, University of Michigan School of Medicine, \\ Ann Arbor, Michigan 48109
}

(Received 17 March 2006; revised 3 May 2006; accepted for publication 25 May 2006; published 12 July 2006)

Photoacoustic tomography (PAT), a nonionizing, noninvasive, laser-based technology was adapted to joint imaging for the first time. Pulsed laser light in the near-infrared region was directed toward a joint with resultant ultrasonic signals recorded and used to reconstruct images that present the optical properties in subsurface joint tissues. The feasibility of this joint imaging system was validated on a Sprague Dawley rat tail model and verified through comparison with histology. With sufficient penetration depth, PAT realized tomographic imaging of a joint as a whole organ noninvasively. Based on the optical contrast, various intra- and extra-articular tissues, including skin, fat, muscle, blood vessels, synovium and bone, were presented successfully in images with satisfactory spatial resolution that was primarily limited by the bandwidth of detected photoacoustic signals rather than optical diffusion as occurs in traditional optical imaging. PAT, with its intrinsic advantages, may provide a unique opportunity to enable the early diagnosis of inflammatory joint disorders, e.g., rheumatoid arthritis, and to monitor therapeutic outcomes with high sensitivity and accuracy. (c) 2006 American Association of Physicists in Medicine. [DOI: 10.1118/1.2214166]

Key words: photoacoustic tomography, inflammatory arthritis, joints

\section{INTRODUCTION}

Inflammatory arthritis encompasses many pathological rheumatic diseases including rheumatoid arthritis (RA) and seronegative spondyloarthropathies. RA, the most common form of inflammatory arthritis, is a systemic disease predominantly manifested in the synovial membrane of diarthrodial joints. ${ }^{1}$ About $1 \%$ of the population is affected by RA and $80 \%$ of the patients are disabled after 20 years. ${ }^{2}$ The synovium affected by RA is marked by neovascularization, inflammatory cell infiltration, and associated synoviocyte hyperplasia. ${ }^{3}$ Synovial membrane inflammation is one of the earliest pathologic changes in RA and other inflammatory joint diseases. Because the enhanced blood vessel growth contributes to the inflammatory joint destruction, inflammatory arthritis is now widely regarded as an angiogenesisdependent disease. ${ }^{4-7}$ Despite the hypervascularization, the rheumatic synovium appears to be a relatively hypoxic environment caused by an imbalance between local metabolic rate and synovial vascular supply. ${ }^{8-11}$

Implementing effective treatments for patients with inflammatory arthritis, i.e., early initiation and optimal adjustments of therapies, requires technologies for early diagnosis and highly sensitive monitoring of disease progression. ${ }^{12}$ Driven by clinical investigations looking for optimized thera- pies and pharmaceutical industries searching for new drugs, musculoskeletal imaging is playing an increasingly important role in the diagnosis, assessment and monitoring of arthritis. Recent advances in joint imaging, including magnetic resonance imaging (MRI) ${ }^{13,14}$ and ultrasonography, ${ }^{15-17}$ have added objective data enhancing diagnosis and treatment monitoring of these diseases. However, identification of initial natural disease sequelae, early diagnosis, and pharmaceutical based intervention and disease progression monitoring still present challenges.

Recently, nonionizing optical modalities for imaging and sensing of joint diseases have drawn considerable attention. Optical imaging of biological tissues is highly desirable because of known intrinsic optical contrast that is sensitive to tissue abnormalities and function. Optical properties of tissue in the visible and near-infrared (NIR) region of the electromagnetic spectrum can demonstrate the molecular constituents of tissues and the electronic or vibrational structures at the molecular scale. Similar to tumors, the hallmarks of rheumatic joint tissues include angiogenesis, hypervascularization, hypermetabolism, hypoxia, and invasion into normal adjacent tissues. Optical properties may be used to quantify these morphological and physiological changes and, consequently, potentially enable the early diagnosis of inflamma- 
tory arthritis and provide improved monitoring of therapeutic interventions with high sensitivity and specificity.

Recent studies have shown that near-infrared spectroscopy (NIRS) can be used to examine the components of synovial fluid and can potentially predict the presence or state of inflammatory arthritis. ${ }^{18,19}$ Based on NIR diffuse optical tomography (DOT), images formed utilizing light absorption and scattering of joint structures of human fingers have been explored. ${ }^{20-22}$ Wavelength-dependent laser CT of human joints has been realized, which can present both structural and functional aspects of joint regions. ${ }^{23}$ Laser based optical tomography for imaging of finger joints has presented the advantages of optical contrast over the existing imaging modalities for early diagnosis and monitoring of inflammatory arthritis. ${ }^{24-27}$ However, due to the overwhelming scattering of light in biological tissues, current optical technologies cannot delineate subsurface joint tissues with satisfactory imaging quality for clinical applications. For example, optical imaging of joints based on DOT cannot achieve spatial resolution better than $5 \mathrm{~mm},{ }^{21}$ insufficient for evaluating the small joint structures of the hands and feet.

Photoacoustic tomography (PAT), also referred to as optoacoustic tomography or thermoacoustic tomography, ${ }^{28-39}$ is a novel hybrid imaging modality that combines high optical contrast and high ultrasonic resolution. In laser-based PAT, a short-pulsed laser source is used to irradiate a biological sample (e.g., joint) with an input energy density less than the safety limit. When light energy is absorbed by the biological tissues, ultrasonic waves are excited due to thermoelastic expansion. The light generated photoacoustic waves are measured by broadband ultrasonic transducer(s) around the sample under study. The ultrasonic transducer is very sensitive in detecting the propagated waves from an absorbing object that are caused by weak absorption of optical energy. Finally, through a reconstruction algorithm, ${ }^{40,41}$ the photoacoustic signals acquired at different positions around the sample are used to rebuild the distribution of optical absorption in the sample, i.e., to map the optical inhomogeneity in biological tissues.

In comparison with traditional optical imaging, the spatial resolution of PAT is not limited by the large scattering of light in biological tissues but instead determined by the detection bandwidth of the transducer and the scanning geometry. As a result, PAT can image tissue structures and functional changes with high spatial resolution (better than 200 micrometer, adjustable with detection bandwidth), which especially favors imaging of the small joint structures of the hands and feet that are usually among the earliest to be affected by rheumatoid arthritis. As with traditional optical tomography, the optical signal employed in PAT to generate ultrasonic waves is sensitive to molecular conformations of biological tissues including both deoxy- and oxyhemoglobin, as well as to soft tissue changes such as hypervascularization. Both abnormal oxygenation state and, as a consequence of increased angiogenesis, hypervascularization are known to occur in inflammatory arthritis. Based on these characteristics along with high intrinsic optical contrast of joint tissues, PAT may provide a unique opportunity to en- able early diagnosis and monitoring of therapeutic interventions in inflammatory arthritis with high sensitivity and specificity.

\section{MATERIALS AND METHODS}

\section{A. Animal samples}

In this study, we explored and optimized the PAT system for joint imaging in the rat model. Rat tail joints provide good samples to study the performance of PAT of human finger or toe joints considering their morphological similarity. Rheumatic disease rat models, including those with inflammatory arthritis, have been researched extensively and provide the opportunity to evaluate pathologic progression much more quickly than in humans. PAT, based on high sensitivity optical signals, provides a potentially powerful tool for the laboratory study of inflammatory arthritis by presenting both morphological and physiological characteristics of joint tissues. As PAT is nonionizing, noninvasive, and with an imaging depth in the NIR region up to several centimeters, enabling penetration of human fingers and toes, ${ }^{39}$ the transition from laboratory prototypical device for animal models to clinical instrument for humans should be accomplished relatively easily and is promising.

Adult Sprague Dawley rats ( $\sim 300$ g, Charles River Laboratory) were included in this study. Whole tails were harvested from the rat bodies within 1 minute after the rats were sacrificed. An electrocautery device (SurgiStat, Valleylab) was then used to clot blood and seal vessels. Before image acquisition, tail hair was removed using hair remover lotion as large amounts can cause light scattering. Each rat tail was placed in the PAT system along the $Z$ axis [see Fig. 1(A)]. The first proximal segment of the rat tail was fixed on a rotational stage that, driven by a stepper motor, could rotate the tail around its axis. The imaged joint was about $2.5 \mathrm{~cm}$ from the rat trunk, where the diameter of the tail was $\sim 8 \mathrm{~mm}$ and the length of a segment was $\sim 10 \mathrm{~mm}$.

After images were recorded, rat tails were saved in $10 \%$ buffered formalin for 3 days. Tails were then decalcified with formic acid for 4-7 days and monitored with a Faxitron MX-20 x-ray system: Once specimen decalcification was completed they were dehydrated with graded alcohol (Hypercenter XP by Shandon), embedded in paraffin (Paraplast Plus), cut into blocks, and sectioned to 7 micron thickness with Reichert-Jung 20/30 metal knife (paraffin microtome). Hematoxylin and Eosin staining of specimen sections on glass slides was conducted. Finally, the histological pictures of specimen sections were taken with a $10 \times$ magnification.

\section{B. PAT system for joints}

The PAT system for joint imaging involves laser pulse generation and delivery, photoacoustic signal reception, and image reconstruction and display, as shown in Fig. 1(A), where an $X-Y-Z$ coordinate system is also indicated. An OPO system (Vibrant B, Opotek) pumped by an Nd:YAG laser (Brilliant B, Bigsky) is used to provide laser pulses with a pulse repetition rate of $10 \mathrm{~Hz}$ and a pulse width of $5.5 \mathrm{~ns}$. In 


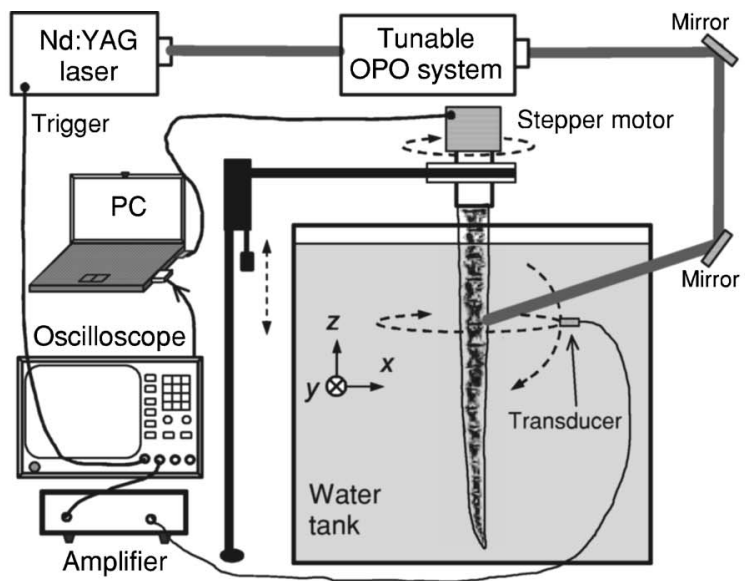

A

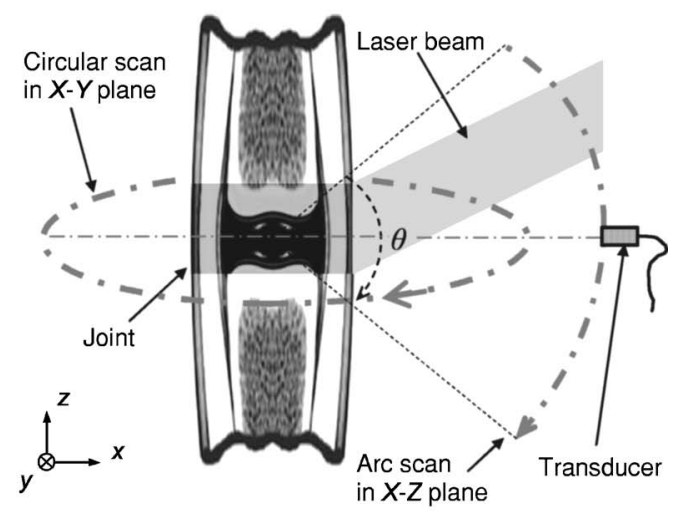

B

FIG. 1. (A) Schematic of the PAT system for joint imaging. (B) An expanded view of the spherical shape scanning geometry around the imaged joint.

this study, the wavelength of the laser light is tuned by the OPO system to $700 \mathrm{~nm}$, which is in the near-infrared region and can enable good penetration depth in biological tissues. The laser beam, after being expanded and homogenized, irradiates the imaged joint with an input energy density of $\sim 15 \mathrm{~mJ} / \mathrm{cm}^{2}$, well below the ANSI safety limit of $20 \mathrm{~mJ} / \mathrm{cm}^{2}$. ${ }^{42}$ The laser light penetrates into the joint tissues and generates photoacoustic signals that are scanned by a wideband ultrasonic transducer (XMS-310, Panametrics) around the joint. This transducer has a central frequency of $10 \mathrm{MHz}$, a full width at half-maximum (FWHM) amplitude bandwidth of $\sim 100 \%$, and an element size of $2 \mathrm{~mm}$ in diameter. To couple the photoacoustic signals, both the sample and the transducer are immersed in a tank of water. After a preamplifier (PR5072, Panametrics), the detected signals are digitized by an oscilloscope (TDS 540B, Tektronics) and then collected by a computer for image reconstruction using a back-projection algorithm. ${ }^{40,41,35}$ For both two-dimensional (2D) and three-dimensional (3D) imaging, the backprojection is performed in the time domain, where the positions of the absorbing objects are determined by the time-offlight and the acoustic velocities in the articular tissues (set as $1.5 \mathrm{~mm} / \mu \mathrm{s}$ in this study). To reconstruct an image, the measured photoacoustic signals are first back-projected into the image space and then integrated over all receiving angles.

To realize $2 \mathrm{D}$ cross sectional imaging of the rat tail joint, the ultrasound transducer can be stepped in a circular fashion around the center of the joint in the $X-Y$ plane, or by rotating the tail axially while keeping the transducer and the laser beam static, as employed in our experiment. To cover a $2 \pi$ receiving angle, 240 steps with a constant $1.5^{\circ}$ interval is conducted, where the radius for the circular scan is $20 \mathrm{~mm}$. With a $10 \mathrm{MHz}$ transducer, the current PAT system for joint imaging with a $100 \%$ bandwidth (spatial frequencies from 3.3 to 10 cycles $/ \mathrm{mm}$ ) exhibits a line spread function (LSF) with a FWHM of less than 250 micrometer in the $X-Y$ plane (Fig. 2). Considering the 50 micrometer size of the line object for the measurement of the LSF, the system spatial reso- lution in the $X-Y$ plane is about 200 micrometer. This spatial frequency response is sufficient to describe many smaller tissue structures in human finger or toe joints, and can be improved further by employing a transducer with a broader bandwidth for signal receiving.

To achieve 3D imaging of a joint, one may move the transducer (or the sample) along the $Z$ axis in Fig. 1 and acquire information to reconstruct $2 \mathrm{D}$ images of multiple cross sections. In this way, although the resolution in the $X$ $Y$ plane is high, the resolution along the $Z$ axis is limited and is primarily dependent on the transducer's $Z$ dimension (i.e., the element size along the $Z$ axis) and the radius of the scanning circle. In order to achieve 3D imaging of a volume with high resolution along each direction, photoacoustic signals need to be collected along a surface, such as a sphere with

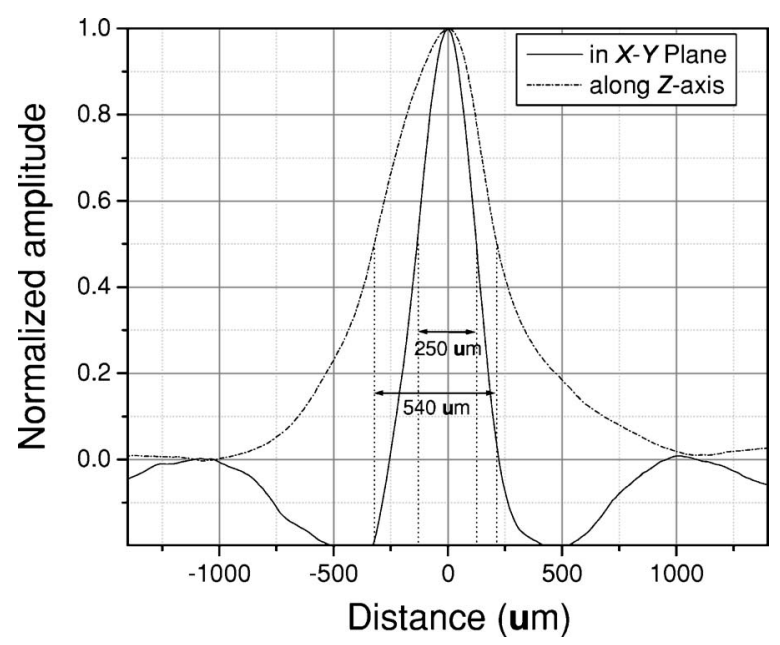

FIG. 2. Measurements of the line spread functions (LSF) of the PAT system in the $X-Y$ plane (solid curve) and along the $Z$ axis (dashed curve). The full width at half-maximum (FWHM) of the LSF in the $X-Y$ plane is $250 \mu \mathrm{m}$; while the FWHM of the LSF along the $Z$ axis is $540 \mu \mathrm{m}$. The line object is a soft tubing filled with high absorbing ink, where the inner diameter of the tubing is $50 \mu \mathrm{m}$. Therefore, the spatial resolution of this system in the $X-Y$ plane is better than $200 \mu \mathrm{m}$; while the spatial resolution of this system along the $Z$ axis is better than $500 \mu \mathrm{m}$. 
two open ends, around the joint. Signals acquired at different positions on the surface are then summed coherently to reconstruct a 3D image of the volume. In this way, the realized synthesized $Z$ aperture will enable high resolution along the $Z$ axis.

To achieve that large nearly spherical synthetic aperture, in this study, besides rotating the tail to realize a circular scan around the joint, the transducer is stepped along an arc that is in a sagittal plane of the tail facing the center of the joint as shown in Fig. 1(B). In the experiment of 3D imaging of rat tail joints, each full circular scan takes 120 steps with a constant radial angular interval of $3^{\circ}$. The arc scan in the sagittal plane takes 24 steps with a constant interval of $3^{\circ}$, which covers an angle $\theta=72^{\circ}$ for a total solid angle of $2.35 \pi$ radians. With this spherical shape scanning geometry, the current PAT system with a $10 \mathrm{MHz}$ transducer can realize a spatial resolution along the $Z$ axis better than 500 micrometer (Fig. 2).

In photoacoustic tomography realized through reconstruction of signals detected by an unfocused ultrasonic transducer, large solid angle for signal detection is essential for good image quality. Therefore, in both $2 \mathrm{D}$ and $3 \mathrm{D}$ experiments on rat tail joints, the transducer scanned around the joint with a large angle for signal receiving. Employing the scanning geometry and reconstruction algorithm described above, the element size of the transducer is another issue that may affect the image quality. The larger the transducer aperture, the more the lateral resolution may be degraded. ${ }^{43} \mathrm{On}$ the other hand, the transducer element size must be big enough to possess sufficient sensitivity for signal detection. In this study, the employed transducer has comparatively high sensitivity and small element size, which also ensured the good performance in imaging small joint structures.

\section{RESULTS}

In the 2D image of an axial cross section of a rat tail joint acquired through a single circular scan around the cross section [Fig. 3(A)], many extra- and intra-articular tissues structures are presented successfully as marked. The spatial resolution achieved by this imaging technique is much better than the results of traditional optical imaging of joints. ${ }^{21,24,27}$ Based on the optical contrast among various tissues, extraand intra-articular joint structures, including skin, fat, muscle, blood vessels, synovium and bone, are described clearly and match well with the histological photograph taken from a similar cross section in the joint [Fig. 3(B)]. The computational time to reconstruct this $2 \mathrm{D}$ image is about 20 seconds using a PC with a $3 \mathrm{GHz}$ Pentium 4 Processor. Another axial 2D photoacoustic image of a joint is shown in Fig. 3(C) with better view of synovium but comparatively lower resolution. The difference in presenting the synovium may be caused by the relative position and orientation of the scanning plane with respect to the imaged joint when 2D imaging was performed. 3D PAT of rat tail joints, based on the scan of the transducer along a spherically shaped surface around the joint, was also performed. The image in Fig. 3(D) shows a $2 \mathrm{D}$ sagittal plane reconstructed from a 3D image of the rat tail joint normal to the slice in Fig. 3(A) and through the line shown therein. Based on the optical contrast, tissues structures in the sagittal section in the joint, notably the synovium, have been presented successfully. The computational time to reconstruct a $3 \mathrm{D}$ joint image is about 56 hours using the $3 \mathrm{GHz}$ Pentium 4 PC.

In both $2 \mathrm{D}$ and $3 \mathrm{D}$ imaging of joints, PAT visualizes the optical absorption distribution in biological tissues that is contributed by various tissue constituents, including water, oxy- and deoxy-hemoglobin, lipid, and mineral. The gray levels in the images present the relative optical absorption in the imaged axial and sagittal sections of the joint, where brighter areas show tissues with comparatively higher optical absorption coefficients. Blood vessels, synovial membrane and bone present relatively higher absorption compared to other surrounding tissues such as fat. These results match those observed by traditional optical imaging of joints. ${ }^{21,22}$ At the $700 \mathrm{~nm}$ wavelength employed in this study, the dominant absorbing material in soft tissues is hemoglobin. Therefore, the presented contrast among soft tissues primarily depicts the hemoglobin concentrations distributed in the joint. We notice that the bone in the joint also shows prominent photoacoustic signal intensity, which is due to not only the intrinsic optical absorption coefficient but also the strong optical scattering in the bone material. ${ }^{44}$

\section{DISCUSSION}

To our knowledge, the adaptation of a photoacoustic technique to noninvasive nonionizing imaging of joints is unique and is completed for the first time. The intrinsic advantages of PAT, namely high optical contrast and good ultrasonic resolution, enable the delineation of small tissue structures in the joints clearly and accurately, with potential for metabolic functional imaging.

Some limitations of the current PAT system include relative lower temporal resolution as scanning is done using a single-element transducer. The signal acquisition time to image one cross section of a joint is $\sim 30 \mathrm{~min}$. In the future, however, with the use of a broadband ultrasonic transducer array and a laser system with a higher repetition rate, it should be possible to use PAT for real-time imaging of joint cross sections while accumulating high resolution 3D data. ${ }^{45,46}$ Moreover, with smaller element size, the ultrasonic transducer array will enable finer scanning and larger acceptance angle for photoacoustic signal detection. The consequently large synthetic aperture will provide higher sensitivity and greatly improved spatial resolution in $3 \mathrm{D}$ imaging of a joint.

Noticeable artifacts can be recognized in current PAT of joints, which, for example, likely causes the synovium in Fig. 3(A) to not be consistently presented. First, image artifact is at least in part a consequence of the limited detection bandwidth of ultrasonic transducer that produces negative values around high absorption targets such as blood vessels. Second, the reflection of photoacoustic signals from bone surface is another potential source of artifact. Finally, acoustic heterogeneity of joint tissues may cause distortion of 

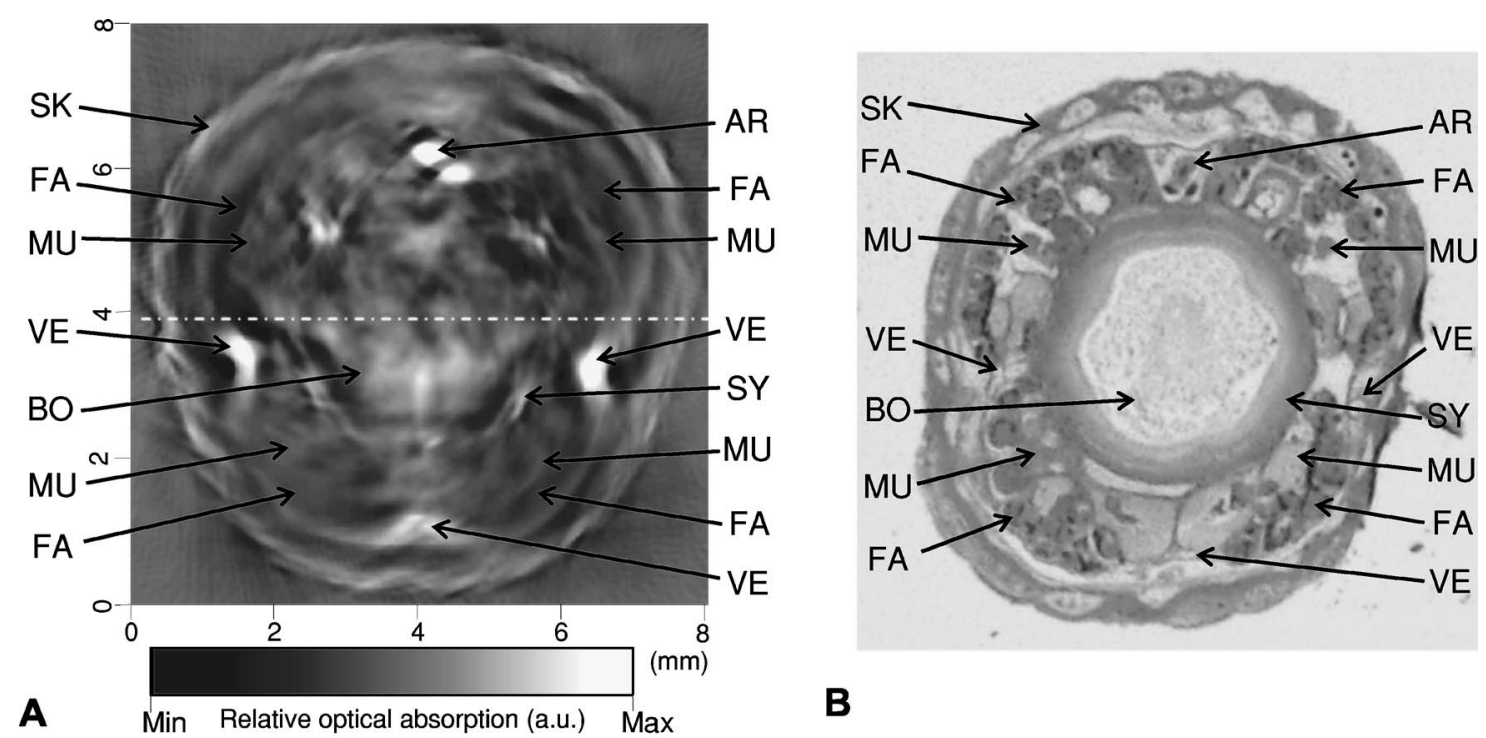

B
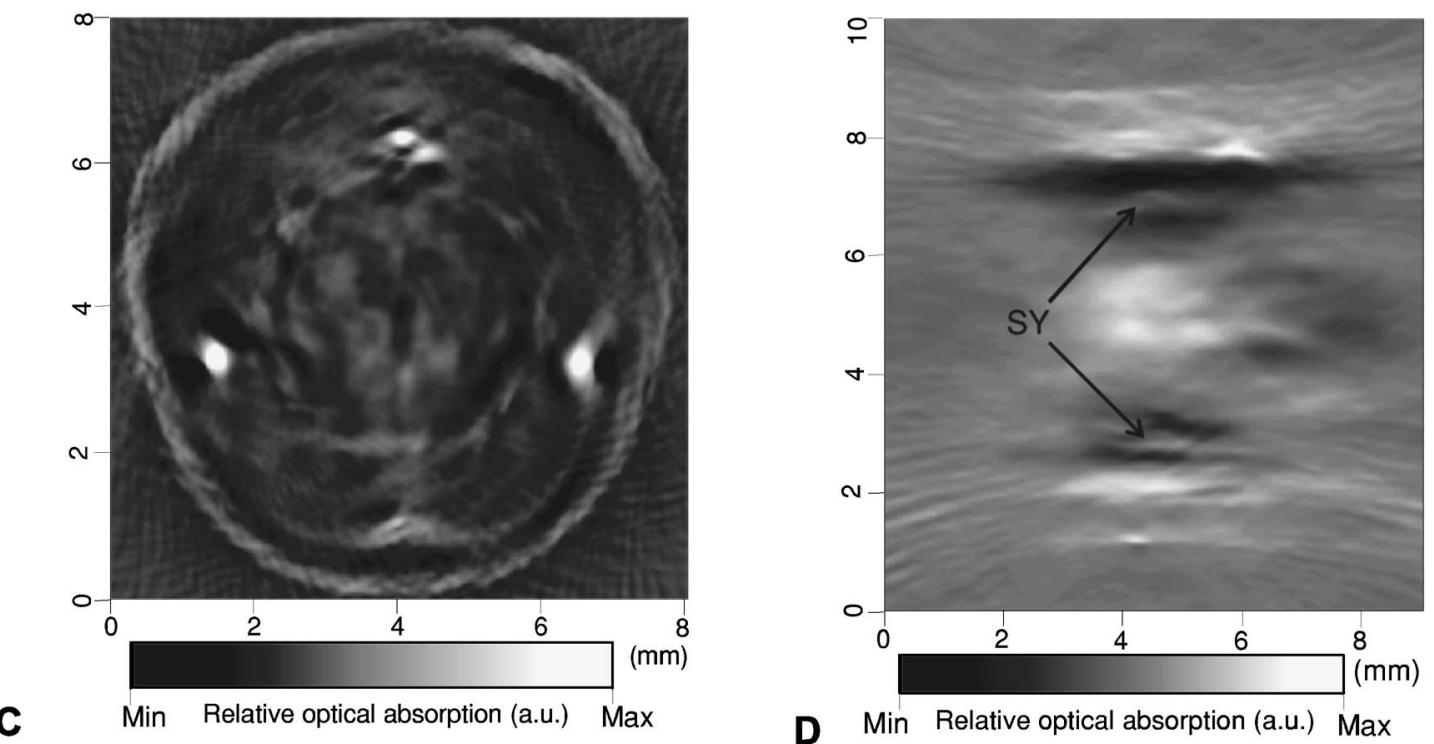

FIG. 3. (A) 2D Noninvasive photoacoustic image of a cross section of a rat tail joint marked with discernable intra- and extra-articular tissue structures. (B) Histological photograph of a cross section of a rat tail joint taken along the plane as closely matched as possible to that of the PAT image. (C) Another 2D cross-sectional image of a joint with better view of synovium. (D) 2D noninvasive photoacoustic image of a sagittal section of a rat tail joint reconstructed from a 3D image volume along the dashed line shown in (A). SK, skin; FA, fat; MU, muscle; VE, vein; BO, bone; AR, artery; SY, synovium.

acoustic wave propagation. Moreover, the inhomogeneous distribution of light fluence in the imaged cross section (or volume in 3D PAT) may also produce unwanted errors in presenting the distribution of optical absorption. Part of our future research will be focused on understanding and minimizing these problems.

With the current PAT system, the same spatial resolution is difficult to realize at depths required for imaging large joints such as human knee joints, though it may be feasible after further system improvement. However it is of note that the potential value of PAT in evaluating joint diseases still holds because peripheral small joints such as those of the feet and hands are usually among the earliest to be affected by rheumatoid arthritis and are widely accepted to be markers of overall joint damage.
Objective quantification of joint pathology is a major challenge for rheumatologists, radiologists, and scientific researchers not only in the clinical setting, but also in research settings where serial assessment of efficacy of new therapies is needed. Presenting high contrast between normal and abnormal tissues in inflamed joints, specifically synovium, bone, muscle, and vessels, PAT can potentially realize accurate mapping and an objective grading of synovitis, enabling a more comprehensive description of the pathologic state of joints. The specific morphologic variables potentially monitored by PAT as biomarkers for inflammatory arthritis include increased angiogenesis and hypervascularization in proliferative joint-associated tissues, synovial hypertrophy and characteristic erosive bone changes. Besides these vascular and joint structural changes, PAT employing multiple 
wavelengths may evaluate hemodynamic changes in joint tissues such as hemoglobin concentration (and, by extrapolation, blood volume) and blood oxygen saturation, which can potentially help to quantify the hyperemia and hypoxia in intra- and extra-articular joint tissues. ${ }^{31,36}$ The high sensitivity of optical signal to these structural and functional hallmarks of synovitis makes PAT a potentially powerful imaging technology with which to study inflammatory joint diseases.

\section{ACKNOWLEDGMENTS}

This research is supported partially by Arthritis National Research Foundation. The authors thank Dr. Jonathan Rubin at University of Michigan and Dr. Lihong Wang at Texas A\&M University for useful discussions and suggestions.

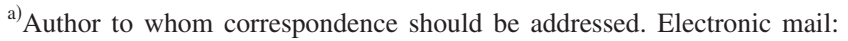
xdwang@umich.edu

${ }^{b)}$ Electronic mail: davicham@med.umich.edu
}

${ }^{1}$ J. H. Klippel (ed.), J. J. Goronzy, C. M. Weyand, R. J. Anderson, and E. L. Matteson, "Rheumatoid arthritis," in Primer on the Rheumatic Diseases, 12th ed., edited by J. H. Klippel (Arthritis Foundation, Atlanta, 2001), pp. 209-232.

${ }^{2}$ D. L. Scott, D. P. Symmons, B. L. Coulton, and A. J. Popert, "Long-term outcome of treating rheumatoid arthritis: results after 20 years," Lancet $\mathbf{1}$, 1108-1111 (1987).

${ }^{3}$ P. E. C. Brenchley, "Antagonizing angiogenesis in rheumatoid arthritis," Ann. Rheum. Dis. 60, iii71-iii74 (2001).

${ }^{4}$ O. FitzGerald, M. Soden, G. Yanni, R. Robinson, and B. Bresnihan, "Morphometric analysis of blood vessels in synovial membranes obtained from clinically affected and unaffected knee joints of patients with rheumatoid arthritis," Ann. Rheum. Dis. 50, $792-796$ (1991).

${ }^{5}$ A. J. Hayes, "Angioneogenesis in rheumatoid arthritis," Lancet 354, 423-424 (1999).

${ }^{6}$ D. A. Walsh, "Angiogenesis and arthritis," Rheumatology 38, 103-112 (1999).

${ }^{7}$ G. S. Firestein, "Starving the synovium: angiogenesis and inflammation in rheumatoid arthritis," J. Clin. Invest. 103, 3-4 (1999).

${ }^{8}$ K. Lund-Olesen, "Oxygen tension in synovial fluids," Arthritis Rheum. 13, 769-776 (1970).

${ }^{9}$ P. S. Treuhaft and D. J. McCarty, "Synovial fluid pH, lactate, oxygen and carbon dioxide partial pressure in various joint diseases," Arthritis Rheum. 14, 475-484 (1971).

${ }^{10}$ J. R. Levick, "Hypoxia and acidosis in chronic inflammatory arthritis; relation to vascular supply and dynamic effusion pressure," J. Rheumatol. 17, 579-582 (1990).

${ }^{11}$ C. R. Stevens, R. B. Williams, A. J. Farrell, and D. R. Blake, "Hypoxia and inflammatory synovitis: observations and speculation," Ann. Rheum. Dis. 50, 124-132 (1991).

${ }^{12}$ F. C. Arnett, S. M. Edworthy, D. A. Bloch, D. J. Mcshane, J. F. Fries, N. S. Cooper, L. A. Healey, S. R. Kaplan, M. H. Liang, H. S. Luthra, T. A. Medsger, D. M. Mitchell, D. H. Neustadt, R. S. Pinals, J. G. Schaller, J. T. Sharp, R. L. Wilder, and G. G. Hunder, "The American Rheumatism Association 1987 revised criteria for the classification of rheumatoid arthritis," Arthritis Rheum. 31, 315-324 (1988).

${ }^{13}$ H. H. Schild, MRI Made Easy (H. Heenemann GmbH and Company, Berlin, 1990).

${ }^{14}$ C. Peterfy, "Imaging techniques," in Rheumatology, 2nd ed., edited by J. Klippen and P. Dieppe (Mosby, Philadelphia, 1997), Vol. 1, pp. 14.114.18.

${ }^{15}$ W. Grassi, "Clinical evaluation versus ultrasonography: who is the winner?," J. Rheumatol. 30, 908-909 (2003).

${ }^{16}$ Z. Karim, R. J. Wakefield, P. G. Conaghan, C. A. Lawson, E. Goh, M. A. Quinn, P. Astin, P. O'Connor, W. W. Gibbon, and P. Emery, "The impact of ultrasonography on diagnosis and management of patients with musculoskeletal conditions," Arthritis Rheum. 44, 2932-2933 (2001).

${ }^{17}$ R. J. Wakefield, A. K. Brown, P. J. O'Connor, and P. Emery, "Power Doppler sonography: improving disease activity assessment in inflamma- tory musculoskeletal disease," Arthritis Rheum. 48, 285-288 (2003).

${ }^{18}$ H. H. Eysel, M. Jackson, A. Nikhulin, R. L. Somorjai, G. T. D. Thomson, and H. H. Mantsch, "A novel diagnostic test for arthritis: multivariate analysis of infrared spectra of synovial fluid," Biospectroscopy 3, 161167 (1997).

${ }^{19}$ J. M. G. Canvin, S. Bernatsky, C. A. Hitchon, M. Jackson, M. G. Sowa, J. R. Mansfield, H. H. Eysel, H. H. Mantsch, and H, S. El-Gabalawy, "Infrared spectroscopy: shedding light on synovitis in patients with rheumatoid arthritis," Rheumatology 42, 76-82 (2003).

${ }^{20} \mathrm{Y}$. Xu, N. Iftimia, and H. Jiang, "Imaging of in vitro and in vivo bones and joints with continuous-wave diffusion optical tomography," Opt. Express 8, 447-451 (2001).

${ }^{21}$ Y. Xu, W. Iftimia, H. Jiang, L. L. Key, and M. B. Bolster, "Threedimensional diffuse optical tomography of bones and joints," J. Biomed. Opt. 7, 88-92 (2002).

${ }^{22}$ J. Beuthan, U. Netz, O. Minet, A. D. Klose, A. H. Hielscher, A. Scheel, J. Henniger, and G. Muller, "Light scattering study of rheumatoid arthritis," Quantum Electron. 32, 945-952 (2002).

${ }^{23}$ B. Devaraj, M. Takeda, M. Kobayashi, M. Usa, K. P. Chan, Y. Watanabe, T. Yuasa, T. Akatsuka, M. Yamada, and H. Inaba, "In vivo laser computed tomographic imaging of human fingers by coherent detection imaging method using different wavelengths in near infrared region," Appl. Phys. Lett. 69, 3671-3673 (1996).

${ }^{24}$ T. Yuasa, S. Tanosaki, M. Takagi, Y. Sasaki, H. Taniguchi, B. Devaraj, and T. Akatsuka, "Transillumination optical sensing for biomedicine and diagnostics: feasibility of early diagnosis for rheumatoid arthritis, Anal. Sci. 17, i515-i518 (2001).

${ }^{25}$ A. K. Scheel, A. Krause, I. M. Rheinbaben, G. Metzger, H. Rost, V. Tresp, P. Mayer, M. Reuss-Borse, and G. A. Muller, "Assessment of proximal finger joint inflammation in patients with rheumatoid arthritis, using a novel laser-based imaging technique," Arthritis Rheum. 46, 1177-1184 (2002).

${ }^{26}$ A. H. Hielscher, A. D. Klose, A, K. Scheel, B. Moa-Anderson, M. Backhaus, U. Netz, and J. Beuthan, "Sagittal laser optical tomography for imaging of rheumatoid finger joints," Phys. Med. Biol. 49, 1147-1163 (2004).

${ }^{27}$ A. K. Scheel, M. Backhaus, A. D. Klose, B. Moa-Anderson, U. J. Netz, K.-G. A. Hermann, J. Beuthan, G. A. Muller, G. R. Burmester, and A. H. Hielscher, "First clinical evaluation of sagittal laser optical tomography for detection of synovitis in arthritic finger joints," Ann. Rheum. Dis. 64, 239-245 (2005).

${ }^{28}$ C. G. A. Hoelen, F, F. M. de Mul, R. Pongers, and A. Dekker, "Threedimensional photoacoustic imaging of blood vessels in tissue," Opt. Lett. 23, 648-650 (1988).

${ }^{29}$ A. A. Oraevsky, S. L. Jacques, R. O. Esenaliev, and F. K. Tittel, "Laser based optoacoustic imaging in biological tissues," Proc. SPIE 2134A, 122-128 (1994).

${ }^{30}$ R. A. Kruger and P. Liu, "Photoacoustic ultrasound reconstruction tomography," Med. Phys. 22, 1605-1609 (1995).

${ }^{31}$ A. A. Oraevsky and A. A. Karabutov, "Optoacoustic tomography," in Biomedical Photonics Handbook, edited by T. Vo-Dinh (CRC, Boca Raton, FL, 2003), Vol. PM125, Chap. 34, pp. 34/1-34/34.

${ }^{32}$ V. G. Andreev, A. A. Karabutov, and A. A. Oraevsky, "Detection of ultrawide-band ultrasound pulses in optoacoustic tomography," IEEE Trans. Ultrason. Ferroelectr. Freq. Control 50, 1383-1390 (2003).

${ }^{33}$ R. G. M. Kolkman, E. Hondebrink, W. Steenbergen, and F. F. M. de Mul, "In vivo photoacoustic imaging of blood vessels using an extreme-narrow aperture sensor," IEEE J. Sel. Top. Quantum Electron. 9, 343-346 (2003).

${ }^{34}$ X. Wang, Y. Pang, G. Ku, X. Xie, G. Stoica, and L. V. Wang, "Noninvasive laser-induced photoacoustic tomography for structural and functional imaging of the brain in vivo," Nat. Biotechnol. 21, 803-806 (2003).

${ }^{35}$ X. Wang, Y. Pang, G. Ku, G. Stoica, and L. V. Wang, “Three-dimensional laser-induced photoacoustic tomography of the mouse brain with the skin and skull intact," Opt. Lett. 28, 1739-1741 (2003).

${ }^{36}$ X. Wang, G. Ku, X. Xie, Y. Wang, G. Stoica, and L. V. Wang, "Noninvasive functional photoacoustic tomography of blood-oxygen saturation in the brain," Proc. SPIE 5320, 69-76 (2004).

${ }^{37}$ X. Wang, G. Ku, M. A. Wegiel, D. J. Bornhop, G. Stoica, and L. V. Wang, "Noninvasive photoacoustic angiography of animal brains in vivo with near-infrared light and an optical contrast agent," Opt. Lett. 29, 730-732 (2004). 
${ }^{38}$ Y. Wang, X. Xie, X. Wang, G. Ku, K. L. Bill, D. P. O’Neal, G. Stoica, and L. V. Wang, "Photoacoustic tomography of a nanoshell contrast agent in the in vivo rat brain," Nano Lett. 4, 1689-1692 (2004).

${ }^{39} \mathrm{G}$. $\mathrm{Ku}$ and L.-H. Wang, "Deeply penetrating photoacoustic tomography in biological tissues enhanced with an optical contrast agent," Opt. Lett. 30, 507-509 (2005).

${ }^{40} \mathrm{M}$. Xu and L.-H. Wang, "Time-domain reconstruction for thermoacoustic tomography in a spherical geometry," IEEE Trans. Med. Imaging 21, 814-822 (2002).

${ }^{41} \mathrm{M}$. Xu and L.-H. Wang, "Universal back-projection algorithm for photoacoustic-computed tomography," Phys. Rev. E 71, 016706 (2005).

${ }^{42}$ American National Standards Institute, American National Standard for the Safe Use of Lasers. Standard Z136.1-2000 (ANSI, New York, 2000).
${ }^{43}$ M. Xu and L. V. Wang, "Analytic explanation of spatial resolution related to bandwidth and detector aperture size in thermoacoustic or photoacoustic reconstruction," Phys. Rev. E 67, 056605 (2003).

${ }^{44}$ A. Pifferi, A. Torricelli, P. Taroni, A. Bassi, E. Chikoidze, E. Giambattistelli, and R. Cubeddu, "Optical biopsy of bone tissue: a step toward the diagnosis of bone pathologies," J. Biomed. Opt. 9, 474-480 (2004).

${ }^{45}$ B. Z. Yin, D. Xing, Y. Wang, Y. G. Zeng, Y. G. Zeng, Y. Tan, and Q. Chen, "Fast photoacoustic imaging system based on 320-element linear transducer array," Phys. Med. Biol. 49, 1339-1346 (2004).

${ }^{46}$ D. W. Yang, D. Xing, H. M. Gu, Y. Tan, and L. M. Zeng, "Fast multielement phase-controlled photoacoustic imaging based on limited-fieldfiltered back-projection algorithm," Appl. Phys. Lett. 87, 194101 (2005). 